\title{
Distorted Cartogram Visualization for Travelers
}

\author{
Jong-Chul Yoon ${ }^{1}$ Jong-Sung Hong ${ }^{2}$ \\ and In Seob Yoon ${ }^{3}$ \\ Dept. of Broadcasting Technology, Kangwon National University 1,2,3 \\ media19@kangwon.ac.kr ${ }^{1}$ jshong@kangwon.ac.kr² isyoon@kangwon.ac.kr ${ }^{3}$
}

\begin{abstract}
Most maps are generated based on measures of distance. However, the time required to reach a points of interest can be more important than the distance for a tourist because it directly indicates availability. We propose a novel geographical visualization technique that represents cartography in terms of the time required to travel between the points of interest rather than the distance. Congestion makes areas of the map expand, whereas ideal traffic conditions make the map shrink in comparison to the actual distance scale of a traditional map. The proposed map visualization application enables a more intuitive scheduling for travelers.
\end{abstract}

Keywords: Cartogram, Geographic Visualization, Web application

\section{Introduction}

Maps provide a simple, yet powerful visualization of geospatial information. Many maps are constructed to representationally reflect geospatial dimensions. Because maps faithfully reflect real-world geography (or try to reflect the three-dimensional Earth as a two dimensional construct as effectively as possible), individuals can intuitively estimate the distance between two points on a map by measuring the distance between them and multiplying this distance by some scale to determine a "real-world" equivalent. The concept of some distance between two points is quite naturally related to the cognitive notion of spatial availability. The proximity of two places is strongly related to how accessible they are from each other; therefore, the construction of a two-dimensional map based on spatial references effectively relates accessibility between places to the user. Unfortunately, proximity may not always predicate the most efficient accessibility. For representing spatial availability, a more direct metric than distance is time.

In this paper, we introduce a time-based distorted map visualization application for travelers. Travelers need scheduling to see many points of interest (POI) in limited time. As shown in Fig. 1(a), existing maps can be expressed as close to absolute distance without considering of roads or traffic volumes. This type of erroneous distance measurement can lead to a wrong schedule for a traveler (see Figure 1(b)), and it would likely lead to a waste of time. However, our proposed map can realize more stable scheduling is possible because it is stretched based on the actual arrival 
time as shown in Fig. 1(c). For time-based map visualization, we use a classical dimension reduction technique to adjust the position between POIs and we propose a distortion method that maintains the shape of the entire map as much as possible based on the POI positions.

With the growing interest in travel time, many researchers have tried to anticipate traffic situations by using various techniques. As a result, many interesting services have been made available to the public. For example, walk score marks a score for every location based on the travel time to surrounding amenities [1]. Traffigram [2] visualizes isochronous contours from a single position to represent arrival times. However, this study considers only the relation between the starting point and the sample points, and the relation between POIs is difficult to apply. We propose a new visualization technique that overcomes these limitations.

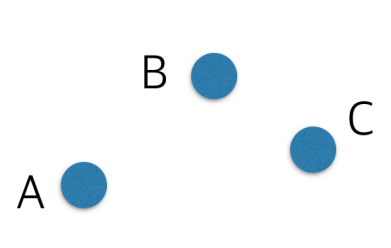

(a)

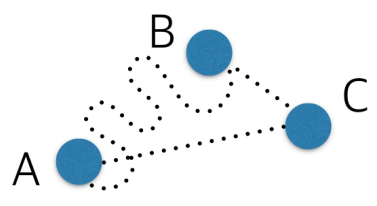

(b)

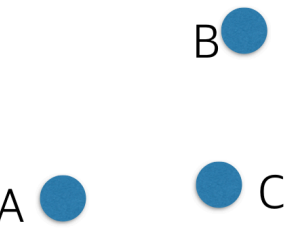

(c)

Figure 1. Concept of time-based distorted map: (a) three POI at the absolute distance based map; (b) road connection information between POI; (c) POI coordinates changed based on arrival time.

\section{Time-based Distortion Map Generation}

\subsection{POI position calculation based on the arrival time}

This study proposes a map distortion method based on each arrival time for a limited number of POIs. We tested this method in the Seattle area. To select POIs, 10 major sightseeing spots provided by TripAdvisor [3] were extracted (Chihuly Garden and Glass, The Museum of Flight, Washington State Ferries, Pike Place Market, Safeco Field, Kerry Park, Sky View Observatory, Space Needle, Benaroya Hall and Hiram M. Chittenden Locks). The travel time between the selected POIs was measured using the Google Maps API. Let the extracted arrival time be $T\left(x_{i}, x_{j}\right)$ which has a symmetric matrix form (where $\mathrm{x}$ is the POI position). We use the classical dimension reduction technique to change the POI position based on the reaching time. Multi-dimensional scaling (MDS) [4] is a traditional dimensional reduction method that has been used in various fields to analyze or visualize nonlinear data sets. MDS is based on the conservation of Euclidian distance in embedded data. If $x_{i}$ is the original position of the POI and $y_{i}$ is the embedded position of the POI based on the arrival time $T(x, y)$, then the objective function for MDS can be descried as follows: 


$$
F=\sum_{i}^{n}\left|T\left(x_{i}, x_{j}\right)-d\left(y_{i}, y_{j}\right)\right|^{2},
$$

where, $d\left(y_{i}, y_{j}\right) \mathrm{r}$ represents the Euclidean distance between the calculated POI positions. By minimizing the change between the arrival time and the Euclidean distance, MDS largely conserves the relationships among the POI positions based on the arrival time. The limitation of the MDS is that the position $y_{i}$, which is changes position according to the metric $T$, may deviate from the original data range and orientation. To solve this problem, we used indiscal [5] as extended dimension reduction technique that solves the MDS problem by placing the four POIs at the outermost points as the constraint. Indiscal is a sequential dimension reduction method that uses weight type constraints. We apply the inverse number of the difference value between the original position and the changed position as the weight of the extracted four extracted POIs to minimize the overall movement.

\subsection{Distorted map visualization}

By using the calculated positions $y_{i}$, we warp the traditional map to the generate a geotemporal cartogram. For this distortion, we use thin-plate spline-based warping (TPS). TPS is a well-known algorithm for various warping applications owing to its low computational cost [6], and it has been widely used as a non-rigid transformation model for image alignment and shape matching. Furthermore, it provides closed-form solutions for interactive-timed warping and it can generate smooth maps while well preserving source images. For implementing this algorithm, we use the Google Maps Javascript API. Furthermore because the existing google map is too complex to represent POI effectively, we design a simple map that expresses representative roads and POI. In addition, in order to make it easier to compare the deformations of existing maps and distorted maps, we visualize the results of TPS warping in grid form, thereby enabling the user to easily grasp the degree of transformation.

\section{Experimental Results and Conclusions}

Figure 2 shows a demo of our proposed system. Figure 2 (a) shows the result of visualization the original POI position on the abstracted map. Figure 2 (b) shows the result of the distorted map based on the arrival time. For a POI located in the city, the results are relatively different owing to the increase in traffic volume and road complexity. POIs on the outskirts of the city tend to have poorer road accessibility. By using these visualization results, the traveler can determine a more efficient order for visiting various destinations. In addition, when a POI is added at the traveler's accommodation location, the arrival time at the starting point can be recognized easily.

Our proposed system uses a distorted map visualization technique based on the time of arrival for multiple POIs. This technique can be used for road planning beyond the purpose of travelers. One limitation of this system is that it cannot check the real-time road traffic volume; instead, it uses the traffic volume from the sampled time. We are currently developing a system that is distorted in real time by using an additional function of Google Maps. In addition, various experiments on the design 
method for map visualization are required, and a verification through a user test will be conducted.

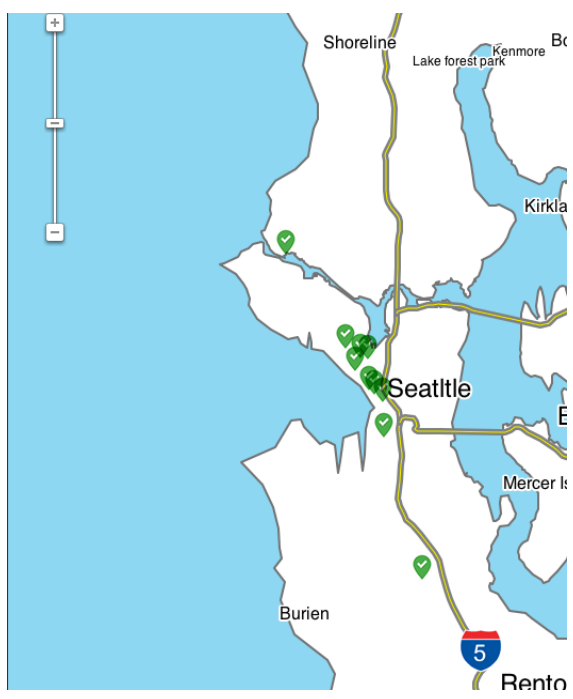

(a)

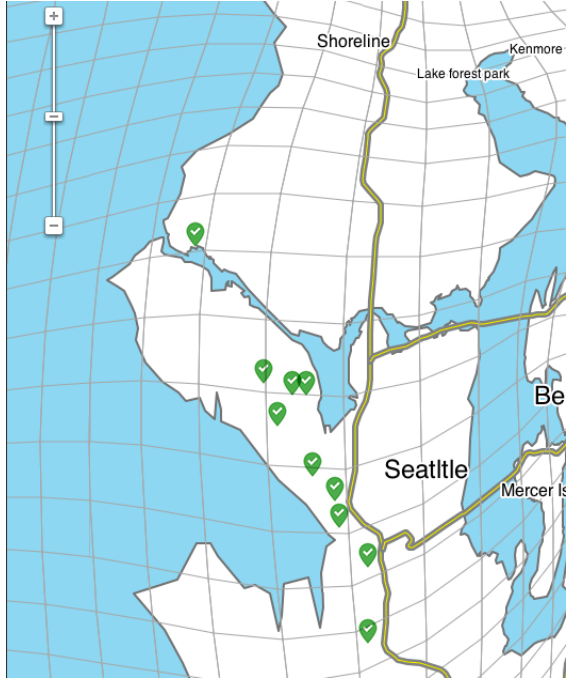

(b)

Figure 2. Time-based distorted map for traveler: (a) abstraction map with ten POI which constructed by original map position; (b) distorted map by TPS warping by using the arrival time between ten POI.

\section{Acknowledgements.}

This research was supported by 2014 Research Grant from Kangwon National University(No. 220140146) and Basic Science Research Program through the National Research Foundation of Korea(NRF) funded by the Ministry of Education, Science and Technology (NRF-2017R1D1A3B03033656).

\section{References}

1. Lightfoot C., Steinberg T.. Travel-time Maps and their Uses. http://www.mysociety.org/ 2006/travel-time- maps. (2006)

2. Sungsoo (Ray) Hong, Yea-Seul Kim , Jong-Chul Yoon, Cecilia R. Aragon, Traffigram: distortion for clarification via isochronal cartography, Proceedings of the SIGCHI Conference on Human Factors in Computing Systems, (2014)

3. https://www.tripadvisor.com (2017)

4. A. K. Jain, R. C. Dubes, Algorithms for Clustering Data, Prentice Hall, (1988)

5. J. R. Barra, F. Brodeau, G. Romier, B. van Cutsem, Recent Developments in Statistics, American Statistical Association, (1978)

6. Wahba, G. Spline Models for Observational Data. Society for Industrial and Applied Mathematics, Philadelphia, (1990) 